\title{
Figement et défigement : problématique théorique
}

\section{Salah Mejri}

\section{(2) OpenEdition}

\section{Journals}

Édition électronique

URL : http://journals.openedition.org/pratiques/2847

DOI : 10.4000 /pratiques. 2847

ISSN : 2425-2042

\section{Éditeur}

Centre de recherche sur les médiations (CREM)

\section{Édition imprimée}

Date de publication : 15 décembre 2013

Pagination : 79-97

\section{Référence électronique}

Salah Mejri, «Figement et défigement : problématique théorique », Pratiques [En ligne], 159-160 | 2013, mis en ligne le 30 juin 2016, consulté le 14 novembre 2019. URL : http://journals.openedition.org/ pratiques/2847; DOI : 10.4000/pratiques.2847 


\title{
Figement et défigement : problématique théorique
}

\author{
Salah Mejri \\ LDI (UMR 7187) \\ Sorbonne Paris Cité-Université Paris 13
}

Les travaux menés ces dernières décennies sur la phraséologie ont focalisé particulièrement sur la notion de figement pour en décrire les mécanismes, les manifestations et le rôle qu'il joue au niveau du fonctionnement des langues. Le défigement, supposé être son corollaire, n'est mentionné qu'accidentellement. Il est le plus souvent défini en creux. Même si certains (Ben Amor 2007, Perrin, à paraître) ont essayé d'en étudier les manifestations discursives ou les outils pragmatiques, il est vraiment rare de trouver des recherches qui en précisent le statut théorique et qui l'installent en tant que concept méthodologique central dans l'étude de la phraséologie. C'est ce que nous comptons faire dans cette contribution. Après avoir rappelé les phases de l'émergence du concept de défigement (en étroite relation avec celui du figement), nous discuterons de la pertinence épistémologique et nous montrerons en quoi il pourrait être à l'origine d'avancées théoriques ${ }^{(1)}$.

\section{L'émergence de la notion de défigement}

Trois phases sont à retenir : une marginalité théorique, une centralité pressentie mais non démontrée et des questionnements théoriques sur le statut du défigement au regard des autres critères du figement.

\subsection{La phase de marginalité}

Il est évident qu'on n'a pas attendu que les linguistes s'occupent du défigement pour qu'on manipule les séquences figées (SF). Comme les SF font partie du lexique des locuteurs, leurs réalisations discursives sont émaillées de SF employées en tant que séquences globales, c'est-à-dire équivalentes aux unités monolexicales. Elles connaissent, comme toutes les autres unités dans le discours, toutes sortes de variations qui peuvent toucher leur intégrité formelle ou sémantique. Selon

(1) Ce travail a été réalisé dans le cadre d'un projet ECOS entre Paris 13 et des institutions mexicaines. 
le contexte, ces manipulations peuvent être volontaires ou involontaires. Dans ce dernier cas, il s'agit le plus souvent soit d'une maîtrise imparfaite des séquences en question (surtout quand il s'agit d'étrangers) soit de lapsus ( $c f$. Freud 1971 et Rossi 1998). Pour les étrangers, il arrive souvent qu'ils retiennent l'intention globale de la séquence sans avoir une maîtrise parfaite des mots exacts qui forment l'unité. On assiste alors à des transformations impliquant des substitutions de mots par d'autres mots faisant partie du même champ sémantique comme en (1):

(1) remuer le couteau dans la blessure (plaie)

ou de prépositions :

(2) travailler dans (à) le noir

Il arrive que la substitution trahisse un déficit lexical quand l'un des constituants n'est pas réalisé correctement : on substitue alors au constituant initial une forme approchée :

(3) prendre la poudre d'estampette (escampette)

Peu importe le mécanisme qui conduit à la formation fautive : le plus important, c'est qu'à l'arrivée, on a une forme qui ne correspond que partiellement à la forme canonique.

Dans le lapsus, les mécanismes à l'origine de la forme réalisée ne sont pas que linguistiques. Des facteurs psychologiques sont à l'œuvre. Ce qui nous intéresse ici, c'est le résultat au niveau de la forme de la séquence :

(4) Merci de votre ascension (attention) (<http://aune.lpl.univ-aix.fr/lpl/personnel/rossi/libre.htm $>$ )

(5) Un professeur d'anatomie cherche à donner une description aussi claire que possible de la cavité nasale qui, on le sait, constitue un chapitre très difficile de l'anatomie du crâne. Lorsqu'il demande si tous les auditeurs ont bien compris ses explications, il reçoit en réponse un oui unanime. A quoi le professeur, connu pour être un personnage fort présomptueux, répond à son tour : «je le crois difficilement, car les personnes qui se font une idée correcte de la structure de la cavité nasale peuvent, même dans une ville comme Vienne, être comptées surun doigt... pardon, je voulais dire sur les doigts d'une main ». (S. Freud, Les lapsus, <http://www.inlibroveritas.net/lire/oeuvre29434-chapitre149750.html>)

Pour ce qui est de la création discursive, il y a lieu de retenir tous les travaux consacrés à la création littéraire et tout ce qu'elle comporte comme versant relatif à la manipulation des SF. L'OuLiPo représente bien cette tendance à exploiter le potentiel de la phraséologie en jouant sur la forme et le sens des SF. Plusieurs travaux sur Queneau montrent 1'ampleur de ce phénomène (Ben Amor, idem). Par ailleurs, toute la littérature ne s'en est jamais privée. Nous fournissons dans ce qui suit deux exemples empruntés à Balzac :

(6) Je suis ravie de voir mon pauvre Felipe tout aussi jeune fille que moi, tout le monde le blesse, il est comme une chauve-souris dans une boutique de cristaux. (H. de Balzac, Mémoires de deux jeunes mariés, Vol. 9, classique Garnier, p. 27.)

(7) - Comment ne vous a-t-il pas offert de vous croquer? dit Bridau. Les peintres sont assez friands de belles personnes.

— Qu'entendez-vous par ces paroles? fit Mme Moreau, sur la figure de laquelle se 
peignait le courroux d'une mine offensée.

- On appelle, en termes d'atelier, croquer une tête, en prendre une esquisse, dit Mistigris d'un air insinuant, et nous ne demandons à croquer que les belles têtes. De là le mot : elle est jolie à croquer !

- J'ignorais l'origine de ce terme, répondit-elle en lançant à Mistigris une œillade pleine de douceur. (H. de Balzac, Un début dans la vie, ibidem, p. 498.)

Ne parlons pas des travaux menés sur le lien entre les jeux de mots et les aspects ludiques. B. de Foucault en dresse une liste assez exhaustive (1988). Nous lui empruntons ces exemples :

(8) «La faim justifie les classes moyennes» (O. Wilde, cité par B. de Foucault, p. 99.)

(9) «Il est malaisé de vivre en bonne intelligence avec les imbéciles » (G. Cesbron, ibidem, p. 105.)

(10) «Je préfère une vraie croyante à une fosse sceptique. » (ibidem, p. 123.)

On peut évoquer également tout ce qui a trait à la traduction des SF et des traces littérales qui accompagnent les tentatives de traduction des SF qui se compliquent indéfiniment avec les séquences défigées ( $c f$. entre autres Équivalences 2008, dont notamment I. Sfar 2008 à propos des blagues et Meta 2008).

\subsection{Une centralité pressentie}

Si dans la première phase les travaux consistaient à défiger les séquences sans chercher à réfléchir sur ce phénomène, un certain nombre de linguistes ont le pressentiment que le défigement représente un aspect central dans l'étude du figement. Déjà G. Gréciano a attiré l'attention depuis 1983 sur le phénomène en rappelant que seul ce qui est figé se défige. G. Gross (1996) est allé plus loin en considérant le défigement comme l'un des critères du figement. Dans un cas comme dans l'autre, la centralité relève du domaine du constat ; le défigement est présenté au même titre que les autres caractéristiques. Quand G. Gross évoque le défigement, il lui attribue le même statut que les dix autres critères retenus pour reconnaître les SF. Cela représente une avancée certaine dans la reconnaissance de ce critère, mais témoigne en même temps d'une période où tous les paramètres du figement connaissent des mutations rapides empêchant un minimum de stabilité pour juger de leur statut théorique. Parmi les événements qui ont marqué cette étape, nous retenons les suivants:

— il y a une remise en question de la notion de figement absolu qui a marqué toute la période antérieure où 1'on croyait que les SF étaient des blocs entiers qui rejetaient toute manipulation. On a même avancé le chiffre de $10 \%$ seulement de la totalité des SF qui répondraient à une telle conception du figement; — on a assisté au retour théorique des descriptions réalisées sur les SF. Les travaux réalisés dans le cadre du LADL et du LLI ont conduit aux conclusions suivantes :

- certains critères employés jusque-là pour reconnaître certaines SF ne sont pas pertinents. Nous rappelons à titre d'exemple le critère de l'absence de déterminant devant le nom des séquences verbales employé par Gougenheim qui a été abandonné parce que des séquences comme avoir peur, avoir faim, avoir froid, etc., tout en partageant l'absence de déterminant, ne font pas partie pour autant de la même catégorie. Si les deux premières sont des constructions à verbes supports, la troisième est une SF ( $c f$. G. Gross 1996); 
- les travaux menés sur les constructions à verbes supports ont montré que tout un pan de ce qui était considéré jusque-là comme des locutions verbales relève, au contraire, de la syntaxe libre et qu'il s'agit en fait de constructions à structure binaire où l'élément verbal ne joue que le rôle d'un actualisateur de l'élément nominal qui est en réalité un nom prédicatif ( $c f$. notamment Giry Schneider 1987);

- découle de ce qui précède l'introduction de la notion de figement relatif qui a pour corollaire celle de degré de figement. Ainsi s'ouvre une nouvelle ère devant les études sur le figement où l'enjeu n'est plus tant la reconnaissance des SF que la mesure de leur degré de figement; ce qui aura un impact théorique très important par la suite, du moment que le passage du figement absolu au figement relatif conduit à un changement radical de la conception même du figement. Avec le figement absolu, il s'agissait de repérer l'ensemble des SF d'une langue pour l'intégrer une fois pour toutes dans le lexique sous forme d'un dictionnaire ; dans la nouvelle perspective, ce programme initial se trouve complètement bouleversé : maintenant, il s'agit de vérifier, moyennant les critères établis, où se situe le figement pour chaque séquence ou pour chaque série de séquences. Ainsi le figement passe-t-il du statut d'une entité à repérer dans sa totalité à celui d'un phénomène diffus qui risque d'être dans certains cas assez ténu pour se confondre avec les contraintes de la syntaxe libre.

Comme l'enjeu, avec ce changement de perspective, n'est plus le même et qu'il s'agit d'évaluer à chaque fois le degré de figement, un retour sur les critères du figement est amorcé chez les uns et les autres pour y mettre un peu d'ordre et en évaluer la pertinence. Parmi les idées avancées dans ce sens, nous en retenons les suivantes :

1. Tous les critères du figement ne doivent pas être mis sur le même plan : les uns relèvent du fonctionnement général de la langue, comme c'est le cas du blocage de la substitution paradigmatique, alors que d'autres ne concernent que la syntaxe propre à certains types de SF : la transformation passive concerne uniquement certaines séquences verbales, l'antéposition de l'adjectif, les séquences nominales ou celles qui comportent dans leurs structures un syntagme nominal, etc.

2. Le degré de pertinence de certains critères de figement admis par la communauté : on commence à s'interroger sur le statut du critère de l'opacité sémantique pour savoir s'il faut le considérer comme un élément définitoire de toute SF ou s'il n'est pas uniquement propre à certaines séquences et pas d'autres. De telles interrogations trouvent leur légitimité dans des faits comme :

- 1'existence d'un très grand nombre de SF transparentes : machine à écrire, fer à repasser, femme de mauvaise vie, etc. ;

- le caractère scalaire de l'opacité sémantique : machine à écrire, fait divers et cordon bleu n'ont pas le même degré d'opacité ou de transparence ;

- le partage de cette gradation par toutes les SF, classées par partie du discours ou ayant une forme phrastique : s'agissant par exemple des proverbes, il est clair que ce sont les formes qui nécessitent une montée synecdochique (G. Kleiber 2013) qui sont opaques; les autres le sont moins ou pas du tout. Comparez (11) et (12):

(11) Qui ne risque rien n'a rien.

(12) Qui vole un œuf vole un bœuf. 
3. L'opacité peut concerner des séquences compositionnelles (Mejri 2011) surtout quand il s'agit de pragmatèmes.

Remise en question de la notion de figement absolu, introduction de celle de continuum dans la variation des SF, retour théorique des descriptions effectuées et discussions de la pertinence des critères appliqués sont les principales raisons qui orientent la réflexion vers une centralité du défigement de plus en plus admise de fait. Reste à préciser la portée théorique du défigement. Devant une telle complexité, plusieurs interrogations s'imposent :

- qu'est-ce qui se défige ? Est-ce la dimension lexicale des SF ? Est-ce la structure syntagmatique? Est-ce l'aspect pragmatique ? Est-ce le croisement de tous ces éléments?

- avec quels outils descriptifs peut-on récupérer les séquences défigées (SD) ? Faut-il récupérer les mêmes moyens utilisés dans la description des SF ou faut-il en forger d'autres et sur quelles bases?

- que faire du statut ambivalent du défigement? S'agit-il d'un phénomène de langue ou d'un phénomène de discours ? Comme les réalisations sont nécessairement discursives, faut-il y voir un phénomène éclaté qui n'obéit qu'à la volonté de celui qui défige, ou au contraire une « grammaire » prévue dans la langue, qu'on peut construire à travers la description de corpus de SD ?

Toutes ces questions ne peuvent avoir de réponse en l'absence d'une réévaluation du statut du défigement au regard des autres critères du figement.

\subsection{Le statut du défigement}

Comme on 1'a souligné plus haut, le défigement n'est utilisé jusque-là que comme un critère parmi les autres critères du figement. Il serait intéressant de voir si ce statut rend compte de la complexité du phénomène ou s'il en fournit une appréciation partielle. Pour ce faire, nous le comparons à l'un ou l'autre des critères du figement.Si nous prenions le blocage de la substitution paradigmatique des constituants de la SF et que nous le comparions au défigement, nous constaterions que le premier, comme le second, se partagent dans la littérature le rôle d'indice de figement : si une séquence connaît un blocage de l'opération de commutation au niveau de ses constituants et si elle donne lieu à un défigement, c'est la preuve qu'elle est figée. Mais on se rend compte rapidement que le défigement dépasse de loin le champ d'action de la commutation. Cela signifie que le déblocage de la commutation des constituants de la SF n'est en réalité que l'une des réalisations possibles du défigement. Le défigement peut s'y limiter comme il peut la combiner à d'autres aspects. Prenons l'exemple suivant :

(13) La tristesse serait donc un abandon à notre nature profonde : nous passons de roseau pensant (selon l'expression de Pascal) à roseau penchant, et penchant plutôt vers le triste... (Christophe André, Les états d'âmes. Un apprentissage de la sérénité, Odile Jacob, 2011, p. 172.)

Le syntagme roseau pensant est défigé grâce à la substitution du participe penchant à pensant (en réalité, il s'agit de la substitution d'un seul phonème), mais le défigement ici ne se limite pas à cette opération, puisque la reprise avec la seconde occurrence penchant permet d'enchaîner le texte avec une forme qui active la valence verbale de penchant, ce qui favorise l'apparition du complément vers le triste. La commutation sert de déclencheur du défigement mais ne couvre pas tout 
l'espace discursif qui lui revient. Dans ce cas précis, l'anaphore fidèle lui échappe. On confond également le défigement avec une autre notion voisine, la variation. Il faut dire que le mot variation n'a pas le statut de terme en bonne et due forme en dehors de ses emplois en sociolinguistique. Or la variation qui nous concerne renvoie aux différentes variantes qu'une SF peut avoir indépendamment de ses dimensions sociolinguistiques. Tout comme les unités monolexicales, elles peuvent avoir deux ou plus de deux formes (signifiants). La différence avec les unités monolexicales, c'est que dans ces dernières la variation porte soit sur 1'orthographe (clé/clef), soit sur la voyelle de jonction entre deux composants (taxinomie/taxonomie), soit sur l'orthographe et la morphologie (trimbalage/trimballage, trimbalement/trimballement), soit encore sur la forme globale (cinéma/ciné) alors que dans les SF, la variation porte sur les mots qui constituent les séquences. Dans les exemples suivants :

(14) ôter/retirer une épine du pied

(15) courir deux/plusieurs lièvres à la fois

(16) rater/louper/manquer le coche

(17) vaincre/battre à plate(s) couture(s)

(18) mettre/avoir la puce à l'oreille

(19) au petit bonheur (la chance)

(20) mettre la charrue devant/avant les bœufs

(21) c'est de l'hébreu/du chinois

(22) être dans la mouise/la panade/la purée

la variation de la même séquence consiste dans la coexistence pour l'un des constituants d'au moins deux formes, ce qui peut concerner n'importe quel type : des verbes $(14,16,17,18)$, des noms (21 et 22), des déterminants (15), ou dans la coexistence d'une forme tronquée et d'une forme complète (19). Derrière de telles variations se profilent des nuances sémantiques, des différences de niveaux de langues ou tout simplement des raisons d'économie linguistique. Qu'il y ait de telles variantes, cela n'affecte en rien le caractère figé des séquences concernées. La différence entre les séquences avec variantes et celles qui n'en ont pas, c'est tout simplement 1'inscription en langue d'unités à choix multiples et d'unités à choix unique. Quand la variation n'assure aucune fonction, l'une des formes disparaît et la séquence à choix multiples rejoint l'autre groupe. Ces proverbes sont souvent employés dans leurs formes tronquées (Mejri 1997) :

(23) Faute de grives, (on mange des merles)

(24) Avec des «si » (on mettrait Paris dans une bouteille)

(25) Eil pour œil, (dent pour dent)

(26) Quand on parle du loup, (on en voit la queue)

(27) Chacun pour soi, (et Dieu pour tous)

(28) Chacun son métier, (et les vaches seront bien gardées)

Si l'on compare ces exemples aux suivants, on saisit la différence entre variation et défigement :

(29) — Eh! Vous nous parlez toujours peinture ! s'écria Georges.

- Ah ! Voilà, chassez le naturel, il revient au jabot, répliqua Mistigris.

(H. de Balzac, Un début dans la vie, ibidem, p. 472.) 
(30) - Tout ce qui reluit n'est pas or, dit-il en lançant des éclairs par les yeux.

— Ça n'est pas ça, s'écria Mistigris. C'est tout ce qui reluit n'est pas fort. Vous n'irez pas très loin en diplomatie si vous ne possédez pas mieux vos proverbes.

- Si je ne sais pas bien mes proverbes, je connais mon chemin.

(H. de Balzac, Un début dans la vie, ibidem, p. 482.)

Deux différences bien saillantes entre les deux notions sont à retenir :

- la variation relève des possibles de la langue, que les locuteurs sont censés connaître, le défigement des possibles du discours, qui, tout en étant contraints par les possibles de la langue, relèvent de l'activité du locuteur ;

- ainsi la variation s'inscrit-elle dans des paradigmes nécessairement fermés alors que le défigement a pour horizons des paradigmes ouverts.C'est en rapport avec ce dernier aspect qu'on envisage de voir la relation entre le défigement et la notion de fixité. Tout comme pour la variation, la notion de fixité ne bénéficie pas d'un statut terminologique clair; elle ne figure pas dans les dictionnaires des sciences du langage, mais se rencontre sous la plume de certains linguistes qui travaillent sur le figement. Elle est souvent utilisée comme une notion définitoire du figement. Est figé dans la langue tout ce qui témoigne d'une fixité (formelle, sémantique ou pragmatique) lors de son emploi dans le discours. Certains lui préfèrent la notion de contrainte. Il nous semble que les deux notions, bien que voisines, ne renvoient pas à la même chose : avec les contraintes, on évoque les limites imposées par l'emploi des unités lors de la réalisation discursive, sans que cela soit spécifique au figement. Elles relèvent des règles de la combinatoire libre. Avec la fixité, on est plutôt du côté de la combinatoire figée, qui impose certes des contraintes dans la partie encore « libre» des SF (dans le cadre du figement relatif), mais gouverne le fonctionnement de la partie totalement figée. Le défigement porte sur des parties ou des aspects fixes des SF. Se défige tout ce qui est fixe. En l'absence de fixité, il n'y a pas lieu de parler de défigement.Cela nous conduit à la question suivante : qu'est-ce qui fait la trame d'une SF ? On peut répondre à cette question par la négative en disant que la trame de la SF serait tout ce qui structure tous les défigements possibles auxquels donnerait lieu cette séquence. Mais cela présuppose qu'on ait une idée précise sur ce qui conditionne les défigements. C'est dans la notion de fixité qu'on pourrait trouver des éléments de réponse. Comme nous l'avons mentionné ailleurs (Mejri 2011), le figement est un phénomène à la fois discontinu et stratifié. La discontinuité repose sur le constat que le figement n'affecte que rarement une séquence d'une manière continue. Dans la plupart des cas, on a soit des séquences admettant des manipulations variées dont l'insertion sépare les constituants des SF, soit des unités pouvant faire l'objet de troncation; ce qui est une autre manière de séquencer les unités polylexicales. Théoriquement, une SF se présenterait selon le schéma suivant :

(31)

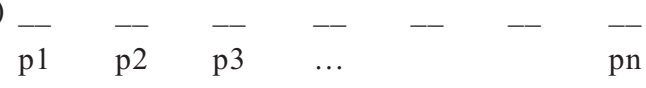

$p$ étant un point du séquençage de l'unité polylexicale.

Mais contrairement à ce que laissent croire le poids du lexical dans l'étude du figement, ces points ne correspondent pas uniquement aux mots entrant dans la formation de la séquence. Comme les SF sont des syntagmes ou des phrases, leur réa- 
lisation nécessite le concours de toutes les composantes du système linguistique allant des phonèmes jusqu'à la dimension pragmatique en passant par la prosodie, la morphologie, la syntaxe et la sémantique. Cette stratification des différents niveaux de structuration des SF conduit à la révision du schéma (31) comme suit, tenant compte de tous les niveaux :

(32) Phonèmes

\begin{tabular}{|c|c|c|c|c|c|}
\hline Phonemes & $\overline{\mathrm{p}^{1}}$ & $\overline{p^{2}}$ & $\overline{\mathrm{p}^{3}}$ & - & $\overline{\mathrm{p}^{\mathrm{n}}}$ \\
\hline Morphèmes & $\overline{\mathrm{mp}}{ }^{1}$ & $\overline{\mathrm{mp}} \mathrm{p}^{2}$ & $\overline{\mathrm{mp}} \mathrm{p}^{3}$ & - & $\overline{\mathrm{mp}} \mathrm{p}^{\mathrm{n}}$ \\
\hline Syllabes & $\overline{\mathrm{s}}{ }^{1}$ & $\overline{\mathrm{sl}^{2}}$ & $\overline{\mathrm{sl}^{3}}$ & - & $\overline{\mathrm{sl}^{\mathrm{n}}}$ \\
\hline Mots & $\overline{\mathrm{m}^{1}}$ & $\overline{\mathrm{m}^{2}}$ & $\overline{\mathrm{m}^{3}}$ & - & $\overline{\mathrm{m}^{\mathrm{n}}}$ \\
\hline $\begin{array}{l}\text { Structures } \\
\text { syntaxiques }\end{array}$ & $\overline{\mathrm{st}^{1}}$ & $\overline{s t^{2}}$ & $\overline{s t^{3}}$ & - & $\overline{\mathrm{st}^{\mathrm{n}}}$ \\
\hline Sens & $\overline{\mathrm{s}^{1}}$ & & $\overline{\mathrm{s}^{3}}$ & & $\overline{\mathrm{s}^{\mathrm{n}}}$ \\
\hline
\end{tabular}

Figure

$$
\overline{\mathrm{f}^{1}} \quad \overline{\mathrm{f}^{2}} \quad \overline{\mathrm{f}^{3}} \quad \bar{\cdots} \quad \overline{\mathrm{f}^{\mathrm{n}}}
$$

Ces différentes strates se croisent entre elles, dans ce sens que chaque point de chaque niveau peut avoir des liens avec tout autre point de n'importe quel autre niveau. Ce croisement de liens assure la globalité de la séquence. Ainsi la globalité serait-elle l'ensemble du croisement des liens entre les différents points de fixité de la trame de la SF. On pourrait lui donner la configuration suivante :

(33) niveau 1

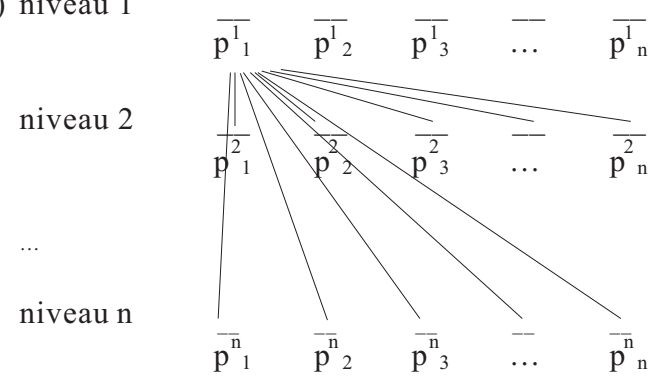

C'est cette solidarité entre tous les points de fixité qui sert de socle à la globalité formelle et sémantique de la SF. Cette trame sert également de base à toute opération de défigement. Comme tous les points sont solidaires, il suffit de toucher un élément pour que le défigement ait lieu. Nous fournissons quelques exemples qui illustrent la pertinence d'une telle structuration :

(34) tout à coup [tutaku]

Cette séquence peut faire l'objet d'un défigement qui ne touche qu'un phonème (un point de fixité). Cette opération annule les liens entre ce point et les points des autres niveaux; ce qui change complètement la configuration de la trame et donne cet effet de méconnaissance de la séquence dont la globalité a été mise de ce fait en question. Si on supprime le troisième phonème [t], ce point aura un impact sur la 
syllabation de la séquence : au lieu de [tu-ta-ku], on aura [tu a ku], ce qui supprime tous les liens établis avec les autres niveaux et donne une configuration nouvelle ne correspondant pas à la trame de la SF.Nous prenons un autre exemple, celui d'un proverbe, où le simple changement d'un phonème ou d'un de ses constituants, [n] au lieu de [r], assure le défigement de la séquence, tout en impliquant les changements sémantiques recherchés par cette opération :

(35) - C'est aussi ta faute à toi, Mistigris, ajouta-t-il en donnant à son rapin une tape sur sa casquette.

— Oh! Moi qui n'ai fait que vous suivre à Venise, répondit Mistigris. Mais qui veut noyer son chien l'accuse de nage! (H. de Balzac, ibidem, p. 488.)

Ce qui est vrai pour les phonèmes l'est également pour les autres niveaux. Contentons-nous de fournir cet autre exemple où ce sont les mots qui sont conservés et où l'effet de défigement est proportionnel aux niveaux impliqués :

(36) Ce n'est pas dans la bouche ou dans le nez que nous trouvons le secret de ce qui est bon à manger ou à boire. La décision du choix revient au cerveau. Mais c'est dans la sphère orale que la qualité de l'aliment est attribuée, étiqueté en quelque sorte « le gosier propose, le cerveau dispose». (Jean-Didier Vincent, Voyage extraordinaire au centre du cerveau, Odile Jacob, 2007, p. 130.)

La désactivation des points de fixité lexicale que sont homme et Dieu auxquels on a substitué respectivement gosier et cerveau implique évidemment les autres niveaux. Dans cet exemple, le niveau métrique est complètement perturbé puisqu'on substitue à la structure binaire 3/3 (lom-pro-poz / dJ $\varphi$-dis-poz) une nouvelle structure binaire 5/5 : (le-go-zjé-pro-poz / le-scr-vo-dis-poz). Il en est de même des phonèmes et des contenus sémantiques.

Après avoir fait ce détour, on est en droit de se demander si le défigement est, comme l'indique toute la littérature, uniquement la réactivation du sens littéral (compositionnel), couplé évidemment à la combinatoire libre qui va de pair. C'est ce que nous essayerons de montrer dans les paragraphes suivants en essayant d'évaluer l'apport heuristique et les avancées théoriques que peuvent réaliser les études sur le défigement.

\section{L'apport heuristique du défigement}

Aborder la question du figement sous cet angle nous permet de reprendre certaines interrogations récurrentes dans la littérature pour en vérifier la pertinence et le fondement méthodologique ; ce qui aboutirait, nous l'espérons, à dévoiler certains aspects non encore traités.

La première question concerne le statut du défigement que nous avons déjà évoqué : serait-il un simple critère du figement? Les éléments que nous avons avancés ne vont pas dans le sens d'une réponse positive à cette question. Même s'il est vrai que c'est un critère qui permet d'identifier les séquences figées, il serait inadéquat de le limiter à ce statut. L'un des arguments qui président à cette position, c'est le caractère dissymétrique de la comparaison qu'on établit souvent entre le défigement et les autres critères. Cette dissymétrie s'explique par l'opposition du défigement à tous les autres critères. Chaque critère du figement a pour rôle de repérer l'aspect de fixité qui le concerne. Si nous prenons le critère de non insertion (G. Gross $1996: 18)$ : 
on constate que ce test permet de montrer le blocage de cette opération, qui est par ailleurs naturelle, dans les combinaisons libres ayant la même structure :

(38) une lumière blanche $\rightarrow$ une lumière très blanche un col vert (canard) $\rightarrow \quad *$ un col très vert

Une telle insertion ne crée pas un défigement relatif uniquement à cette fixité syntaxique mais la dépasse pour toucher d'autres points de fixité, puisque la séquence obtenue, ici un col très vert, n'a plus rien à avoir avec un col vert, dénomination d'un type de canard. Il faut comprendre par là que tous les autres aspects, formels et sémantiques, s'en trouvent touchés. Pour résumer, on peut dire que chaque critère se limite au domaine dont il relève alors que le défigement est par définition global : la séquence défigée est complètement «liquéfiée »; donc tous les points de fixité constituant la trame de la séquence sont touchés par le défigement.

Il s'ensuit que le défigement s'oppose aux critères du figement en s'en servant comme outils et les englobe, par conséquent, dans le résultat auquel il donne lieu. Pour mieux saisir cette relation d'inclusion entre les deux, prenons cet exemple:

(39) Je me suis ouvert le pouce en réparant le pneu crevé du vélo de Grégoire [...] Comme nous étions dimanche, Grégoire m'a proposé de me rendre chez le père de son copain Alexandre, médecin de son état [...] Le docteur pique une première fois, passe le fil, pique une deuxième fois, à la troisième je m'évanouis [...] Sur le chemin du retour, Grégoire m'annonce sa décision de « faire docteur » quand il sera grand. Comme je lui demande les raisons de cette vocation subite, il répond: parce que je ne veux pas que tu meures. Sa réponse, bien sûr, me va droit au cœur où elle atténue les battements de mon pouce. (Il serait plus juste d'écrire : me va droit au pouce où elle atténue les battements de mon cœur.) Ah ! La joie de l'adulte revenu de tout devant la candeur d'une affection enfantine ! En y songeant ce soir, la joie vire au chagrin, celui-là même qu'éprouvera Grégoire au-dessus de ma tombe quand il maudira l'impuissance de son art [...] (Daniel Pennac, Journal d'un corps, Gallimard, 2012, p. 275-276.)

où l'auteur procède in presentia au défigement des deux séquences aller droit au cœur et les battements du cœur. Le fait que cette collocation fasse l'objet d'un défigement est la preuve d'un nombre de points de fixité, donc d'un certain degré de figement. Le procédé utilisé consiste à substituer dans une structure chiasmatique au mot cœur le mot pouce. Le mot pouce renvoie bien au pouce du personnage que le médecin venait de suturer alors que la première occurrence de cœur ne renvoie pas à l'organe ; elle fait partie de la séquence figée aller droit au cour dont la signification globale est «déterminer une émotion ». La seconde occurrence de cœur permet d'avoir une collocation qui se substitue au syntagme incongruent les battements de mon pouce. Comme on le constate, il n'y a qu'une seule opération, la substitution d'un constituant de séquence figée ; pourtant le défigement est total : il touche tous les aspects, formels et sémantiques, des séquences. La preuve en est le caractère incongruent des deux autres séquences obtenues par substitution : les battements de mon pouce et aller droit au pouce.

Cette opposition entre le défigement, phénomène englobant, et les manipulations mises en œuvre pour le réaliser, se voit plus clairement quand il s'agit de la traduction littérale d'une séquence figée d'une autre langue comme le fait T. Ben Jelloun dans le passage suivant : 
(40) $[\ldots]$ Le peintre passa l'une des nuits les plus horribles de sa vie. Le matelas était couvert d'un plastique sur lequel on mettait des draps rêches. Il s'en dégageait une chaleur insupportable. Ça le torturait mais il ne pouvait pas changer de lit. Et s'il s'asseyait, il devait le faire avec d'infinies précautions car il risquait d'arracher les tubes à oxygène par lesquels il respirait [...] Il se souvint de ce que disait sa mère quand elle passait une mauvaise nuit : « cette nuit fait partie de celle que je raconterai à mon fossoyeur $»$. Il riait parce que enfant il ne comprenait pas comment un mort pouvait encore parler et en particulier à son fossoyeur. Et puis qu'allait-elle lui dire ? Qu'elle avait mal dormi, qu'elle avait eu des angoisses, des sueurs froides, une impression de mort imminente avec son cortège de souffrances et d'incertitudes?»(Tahar Ben Jelloun, Le bonheur conjugal, Gallimard, 2012, p. 82-83.)

La séquence [nahki:ha: lhaffa:r qabri:] traduite en français, tout en restant transparente grâce aux éléments contextuels, se trouve versée dans une littéralité qui sert de prétexte à l'auteur pour construire l'ensemble des commentaires portant sur le caractère étrange d'une telle expression. Ainsi aucun aspect n'échappe-t-il au défigement. Le défigement déstructure totalement la séquence figée, même si l'origine de l'opération peut n'être qu'une seule manipulation.

On peut s'interroger sur l'origine de cette dissymétrie entre une manipulation locale et un défigement global. Cela s'expliquerait entre autres par la correspondance entre deux dualités : 1'une est structurelle dans les séquences figées, à savoir le fonctionnement global qui s'oppose au fonctionnement « littéral », non global; l'autre concerne le défigement qui est, lui également, de nature duale : il relève à la fois de la langue (ce qui sert de repère au locuteur : les points de fixité) et du discours (la réalisation discursive).

L'opposition globalité/déglobalité sert d'espace interprétatif à toute séquence défigée. Toute interprétation de telles réalisations discursives ne peut se faire que si on fait le va-et-vient entre ces deux repères délimitant l'espace sémantique de telles séquences. Dans l'exemple suivant :

(41) Si vous avez les yeux plus gros que le ventre, vous n'êtes pas près de trouver une paire de lunettes. (Pierre Dac, Avec mes meilleures pensées, Le Cherche midi, 2010, p. 116.)

l'expression avoir les yeux plus gros que le ventre est interprété d'abord en tant que SF signifiant « avoir plus d'appétit apparent que réel ; être incapable de manger autant qu'on le désirait » (A. Rey et alii, 1989). Mais avec l'apodose comportant une relation méronymique, on assiste à une réactivation du sens littéral. Cette dualité sémantique, qui n'est pas à confondre avec la polysémie des séquences figées, fait le pendant direct de la dualité du défigement qui relève à la fois de la langue (tous les points de fixité formels et sémantiques) et du discours (toutes les actualisations possibles dans les réalisations discursives).

Le défigement, comme l'a si bien démontré L. Perrin (à paraître), a pour support la littéralité de la séquence, indépendamment de la réalité étymologique de l'expression défigée. C'est pourquoi, le défigement offre un grand potentiel discursif, surtout quand les séquences sont construites sur des métaphores et des métonymies, comme l'illustrent ces exemples :

(42) Velouté, tu me mets la pulpe à la bouche. (Publicité de Velouté à la pulpe de fraise. Danone. M. Fr. 6, 81, cité par M.-B. Vittoz Canuto, A. G. Nizet, Paris, 1983, p. 112.) 
(43) Entre chien et grand méchant loup (N. Obs. 28/11/81 (à propos de Modiano et de Robbe-Grillet au royaume des images), cité par Vittoz Canuto, ibidem p. 114.)

Dans l'exemple (43), les deux points de fixité figuratifs sont chien et loup. Le défigement active la littérarité de ces deux items, ce qui fait basculer l'unité polylexicale du côté de la littéralité d'interprétation. Aussi faut-il préciser que le sens global, adossé à la globalité formelle, ne disparaît en aucune façon. L'interprétation littérale est tout simplement mise plus en saillance par rapport au sens global. Les deux significations se font écho d'une certaine manière : l'une ne va pas sans l'autre. D'où cette densité sémantique, souvent exploitée par la publicité, les journalistes et les humoristes. Dans la communication audio-visuelle, les journalistes ont souvent recours à des images pour défiger les expressions ; les caricaturistes également. Par les jeux de substitution, la littérarité crée de nouvelles images adaptées aux exigences de la narration. Dans ce passage de Céline, l'auteur substitue Seine à Rubicond et offre ainsi l'image du personnage hésitant et craintif. Aussi faut-il préciser que l'auteur a recours également, entre autres, à des points de fixité historique, inférée par l'origine de la séquence ( $c f$. l'allusion à César) :

(44) J'aurais été content de ne jamais retourner à Rancy. Depuis ce matin même que j'étais parti de là-bas j'avais presque oublié déjà mes soucis ordinaires ; ils y étaient incrustés si fort qu'ils ne me suivaient pas [...] c'étaient des soucis de banlieue. Cependant vers la rue Bonaparte, la réflexion me revint, la triste. C'est une rue pourtant qui donnerait plutôt du plaisir au passant. Il en est peu d'aussi bienveillantes et gracieuses. Mais, en m'approchant des quais, je devenais tout de même craintif. Je rôdais. Je ne pouvais me résoudre à franchir la Seine. Tout le monde n'est pas César ! De l'autre côté, sur l'autre rive, commençaient mes ennuis. Je me réservais d'attendre ainsi de ce côté gauche jusqu'à la nuit. C'est toujours heures de soleil gagnées, que je me disais. (Céline, Voyage au bout de la nuit, Gallimard, Folio, 1952, p. 287.)

Comme on le constate, il y a un intérêt heuristique certain dans l'étude du défigement : on apprend ainsi que tout est fait dans la langue pour signifier. Les points de fixité repérés à tous les niveaux formels et sémantiques concourent à la production du sens en langue et en discours : phonèmes, syllabes, morphèmes, mots, syntagmes, tropes, rythme, connotations, inférences de toutes sortes. Le défigement exploite toutes ces dimensions pour créer du sens à partir de ce qui est fixé par le figement et qui sert de point de départ à toute manipulation formelle ou sémantique de la trame de la séquence figée.

\section{Les avancées théoriques}

Arrivé à ce point, il serait légitime de se poser la question des avancées théoriques des études sur le défigement. C'est bien d'avoir procédé à sa description. Encore faut-il montrer en quoi de tels travaux font avancer la réflexion sur le figement. Trois points retiennent à ce titre notre attention : le statut du figement par rapport au défigement, le discours comme point de départ et d'arrivée du figement et la reconnaissance des séquences figées.

Pour ce qui est du premier, il y a lieu de souligner que le couple figement/défigement est une dichotomie de base qui fait que l'on ne peut pas étudier l'un sans l'autre et qui sert de support à des liens organiques entre les deux termes de la dichotomie. Le figement porte en lui-même tout le potentiel du défigement : chacun des points de la fixité décrits plus haut peut donner lieu à une manipulation qui peut activer la littéralité de la séquence ou tout ce qui est inféré par les éléments glo- 
baux ou constitutifs de la séquence figée. Ainsi est-on loin de la vision qui voit dans le défigement un simple test de reconnaissance des séquences figées.

Si le figement porte en lui-même les éléments potentiellement susceptibles d'être défigés, le défigement serait alors un élément par lequel on prouve le figement, pas l'inverse. Toute séquence figée n'est pas nécessairement défigée, même si elle l'est potentiellement, mais celles qui le sont fournissent la preuve de leur figement, ou même, encore mieux, le degré de leur figement. Comme seul ce qui est figé se défige, tout ce qui échappe au défigement peut servir de preuve qu'il relève de la combinatoire libre, pas figée. Le meilleur exemple, ce sont les variantes des séquences : leur emploi ne provoque aucun défigement. Remarquez cette ancienne variante de donner la chair de poule employée par Balzac :

(45) Elle m'a doucement attirée sur elle et baisée au front en m'y mettant ce feu qui dévore, qui a noirci l'azur de ses yeux, attendri ses paupières, ridé ses tempes dorées et jauni son beau visage. Elle m'a donné la peau de poule. Avant de répondre, je lui ai baisé les mains. (Balzac, Mémoires de deux jeunes mariées, ibidem, p. 159.)

Peut-être faudrait-il souligner le caractère économique du défigement : il offre des possibilités ouvertes, non finies, d'expressions discursives.

Pour le deuxième point, relatif au discours, il faut rappeler qu'avec les études du défigement, on a réussi à montrer comment ce phénomène établit un pont entre la langue et la parole (le discours), en ce sens qu'il fixe dans le lexique commun des bribes de discours (syntagmes, phrases, etc.) et que ces mêmes unités se retrouvent réinvesties de nouveau dans le discours comme unités lexicales. Un tel processus, celui du figement, se fait selon des opérations complexes de conceptualisation et de globalisation, qui vont de la littérarité des syntagmes libres de départ aux unités polylexicales, dont le fonctionnement formel et sémantique concerne la globalité de l'unité.

Avec le défigement, le discours sert d'espace dans lequel le mouvement inverse s'opère : de la globalité formelle et sémantique, la séquence figée est orientée vers la littéralité. Cela se fait à la faveur de l'ancrage de l'unité polylexicale dans un espace discursif dont elle sélectionne des éléments servant de points d'activation de sa littéralité. Dans le passage suivant de Céline, on remarque comment le proverbe qui vole un æuf vole un bæuf, qui signifie « on commence par de menus larcins, puis on commet des vols plus importants » (A. Rey et alii, idem), fait l'objet de remaniements formels permettant d'ajouter à sa structure binaire un troisième élément renforçant la gradation signifiée initialement par le proverbe :

(46) Quand je n'étais pas à moisir de fièvre sur mon « démontable», ou à battre mon briquet primitif, je ne pensais plus qu'aux comptes de la «Pordurière». C'est curieux comme on a du mal à s'affranchir de la terreur des comptes irréguliers. Certainement, je devais tenir cette terreur de ma mère qui m'avait contaminé avec sa tradition : «On vole un bœuf... Et puis un bœuf, et puis on finit par assassiner sa mère ». Ces choses-là on a bien du mal à s'en débarrasser. On les a apprises trop petit et elles viennent vous terrifier sans recours, plus tard, dans les grands moments. » (Céline, ibidem, p. 175.)

La manière dont Céline a tourné le proverbe se présente comme suit :

— suppression de la construction corrélée en qui : qui...qui...;

- effacement du pronom qui ;

— substitution de la coordination à la corrélation, marqué par les coordonnants et puis ; 
— ajout d'une nouvelle expansion exprimant l'extrême limite à laquelle aboutirait une telle progression, ce troisième segment comportant un élément anaphorique, mère.

Cette forme défigée sert de cataphore à tout le commentaire qui suit. Cette opération de déconstruction se fait également dans le sens de la complexité syntaxique :

(47) Laisser aboyer les chiens pendant que la caravane passe, c'est prendre le risque de réveiller le quartier jusqu'à ce qu'elle soit passée. (Pierre Dac, ibidem, p. 202.)

L'auteur, dans cet exemple, a substitué à la juxtaposition (les chiens aboient, la caravane passe) une subordination inférée dans la forme canonique, sans toucher à l'essentiel des éléments de la protase et de l'apodose, qui se trouvent réactivés par leurs reprises par la suite.

Il est évident que le défigement peut s'opérer sans la moindre altération du signifiant de surface :

(48) L'habit ne fait pas le moine, mais le chapeau fait l'évêque et les quatre points, les cardinaux. (P. Dac, ibidem, p. 195.)

Si on fait abstraction des points cardinaux, il est clair que l'habit ne fait pas le moine se présente ici dans l'intégralité de son signifiant. C'est le contexte droit qui, par le biais de chapeau et évêque (et cardinaux), est à l'origine de l'activation du sens littéral d'habit et moine.

Si dans les exemples précédents la séquence figée est reléguée au second plan tout en servant de référence fondamentale pour l'interprétation du message, dans l'exemple suivant portant la même SF que (48), les deux significations, littérale et globale, coexistent dans une ambiguïté parfaite :

(49) Le lendemain matin, Can me vêtit, m'adouba, un peu comme devaient le faire tant d'années plus tard les jeunes habilleuses de l'Air Force israélienne qui me sanglaient de la combinaison anti-g. Celle-ci me changerait en pilote de chasse, l'habit de moine, tandis que nous m'examinions tous deux dans la glace de mon armoire, faisait un moine. Nul doute, j'étais un jeune abbé, grave, doux, et pénétré, mon missel à la main plus un gros cahier dont j'avais fait l'emplette au dernier instant afin d'y inscrire le nom des bienfaitrices, qui donneraient une pièce, un billet peut-être, pour l'institution des sourds et muets de la rue St-Jacques que j'allais prétendre représenter. Il était manifestement trop tard pour reculer. (Claude Lanzmann, Le lièvre de Patagonie, Gallimard, 2009, p. 153.)

Le déguisement du personnage en moine donnait un résultat vraisemblable : la forme affirmative sollicite la forme négative de la SF sans la contredire, comme c'est indiqué dans la suite du texte (le déguisement est soupçonné par l'une des habitantes du quartier sollicitée pour l'aumône).

C'est cette capacité à structurer le discours qu'il faut également souligner : par le biais du défigement, l'ancrage discursif se fait par toutes sortes de moyens, mais dans tous les cas, des liens cata/anaphoriques s'établissent entre une forme déconstruite (à reconstruire) à partir des éléments minimaux de reconnaissance et une nouvelle forme discursive, apparentée à la première, porteuse d'une signification littérale, propre au discours en question.

Ainsi pourrions-nous évoquer un dernier point, celui de la reconnaissance des 
séquences figées. Nous voudrions dire par là que le défigement peut également permettre de répondre à la question suivante : quels sont les éléments de discours sur lesquels les locuteurs s'appuieraient pour savoir que derrière les formes discursives en question se profilent des SF sans lesquelles l'interprétation des segments du discours ne serait pas possible ou serait amputée d'une dimension essentielle ? En d'autres termes, quels seraient les éléments irréductibles à toute séquence figée, que le défigement doit préserver pour que la dualité sémantique et formelle soit garantie?

Il y a évidemment des séquences figées dont l'actualisation discursive ne risque pas de prêter à confusion. Nous pensons particulièrement à celles qui comportent une partie stable (figée) et une autre variable. Là il ne s'agit pas de défigement et la séquence se reconnaît grâce à sa partie stable. La partie variable se charge de l'actualisation. La séquence et que je te $+v_{e r b} e^{(2)}$ se trouve réalisée dans le discours de différentes manières :

(50) On peut dire qu'on a eu alors de la fête plein les yeux ! Et plein la tête aussi! Bim et Boum ! Et Boum encore ! Et que je te tourne! Et que je t'emporte ! Et que je te chahute ! Et nous voilà tous dans la mêlée, avec des lumières, du boucan, et de tout ! Et en avant pour l'adresse et la rigolade ! Zim ! (Céline, ibidem, p. 447.)

(51) Robinson monte donc derrière avec Madelon dans un baquet et moi dans un autre devant avec Sophie, et on s'en colle une série de fameuses collisions ! Et je te cabosse ! Et je te cramponne! Mais je vois tout de suite qu'elle n'aime pas ça qu'on la bouscule Madelon. Lui non plus d'ailleurs Léon, il n'aime plus ça. On peut dire qu'il n'est plus à son aise avec nous. (Céline, ibidem, p. 478.)

Mais elle ne peut nous servir d'exemple pour montrer comment, malgré une légère différence entre les deux types d'occurrences en (50) et (51), on reconnaît la même tournure. Cela se fait ici à travers trois moyens :

— les éléments lexicaux qui se répètent dans la même structure : et qui ouvre la séquence, que exclamatif, un je qui renvoie à un $i l$ et un te "datif éthique " (Soutet, idem.) [Et que je te] ;

— un élément verbal variable faisant l'objet d'un paradigme lexical ;

— une interprétation de l'« énoncé comme conclusif d'une séquence textuelle complexe » (Soutet, idem.), doublé d'une idée d'intensité.

En d'autres termes, trois types d'éléments se croisent : une structure syntaxique, un lexique (même s'il est de nature grammaticale ici : et, que, je, te) et un rythme. Comme on le constate, on retrouve les fameux points de fixité.

Est-ce que le même processus est engagé dans le cas du défigement? La réponse est oui, mais tout dépend du degré d'altérations formelles et sémantiques portées à la séquence figée. Prenons les exemples suivants :

(52) Toutefois, cette anarchie bien virulente se trouvait renfermée dans un cadre de police hermétique, comme les crabes dans leur panier. Ils bavaient en vain des fonctionnaires, et le Gouverneur trouvait d'ailleurs à recruter pour maintenir sa colonie en obédience, tous les miliciens miteux dont il avait besoin, autant de nègres

(2) $C f$. 1'excellente étude que O. Soutet (2010) a consacré à cette tournure. 
endettés que la misère chassait par milliers vers la côte, vaincus du commerce, venus à la recherche d'une soupe. (Céline, ibidem, p. 126.)

(53) Deux juifs se rencontrent en wagon dans une station de Galicie. « Où vas-tu ? » dit l'un — «À Cracovie.», dit l'autre — «Vois quel menteur tu fais!» s'exclame l'autre. «Tu dis que tu vas à Cracovie pour que je croie que tu vas à Lemberg. Mais je sais bien que tu vas vraiment à Cracovie. Pourquoi alors mentir?» (Freud, cité par Catherine Wieder 2002, p. 116.)

(54) Écrire, c'est comme le métro. Vous savez où vous allez, vous n'avez pas un choix infini de destinations, il y a des horaires à respecter [...] Il y a tout ce que vous ne pouvez pas prévoir [...] Les regards échangés, parfois glissant sur le bouclier des glaces [...] Et les nuques, ah les nuques. On ne parlera jamais assez des nuques [...] Et plus que tout, les pieds. Non pas bêtes, ni beaux, ni glorieux même s'ils sont chaussés de cuir souple, ornés de boucles et d'œillets. Les pieds tout simplement posés sur le sol [...] (J.-M. G. Le Clézio, Histoire du pied et autres fantaisies, Gallimard, 2011, p. 333-334.)

Ces trois exemples ont la particularité de faire se désintégrer la structure syntaxique des séquences figées :

- c'est un panier de crabes $\rightarrow$ les crabes dans leur panier

- aller à Cracovie $\quad \rightarrow \quad$ - Où est-ce que tu vas ? - À Cracovie

— bête comme ses pieds $\rightarrow$ les pieds. Non pas bêtes

En plus des éléments contextuels, on a recours ici aux constituants lexicaux pour reconstituer les séquences figées qui se profilent derrière ces emplois. Dans l'exemple (53), la reprise par la suite de la même séquence aller à Cracovie dans le sens global de " mentir » et dans son sens libre (aller vraiment à Cracovie) confirme le défigement. Mais dans cet exemple, comme dans les deux autres, le contexte assure une congruence sémantique avec la SF concernée : en (52), toute la description qui suit les crabes dans leur panier renvoie en même temps au sens littéral (baver) et au sens global «groupe de personnes qui cherchent mutuellement à se nuire, à se combattre » (A. Rey et alii, ibidem). En (54), il y a négation du présupposé de la littéralité de la séquence intensive bête comme ses pieds.

La reconstitution des séquences figées en question se fait donc à partir des éléments sémantiques contextuels et des éléments lexicaux : on remonte donc du lexique et du sens vers la structure syntaxique. Ailleurs, on intervertit l'ordre, etc.

Ainsi, nous pourrions dire que le défigement s'opère de différentes sortes, et ce en fonction des points de fixité concernés et de leur désactivation ; ce qui peut être ramené en termes de trames aux schémas suivants:

1. Séquence figée : tous les points de fixité activés - sens global :

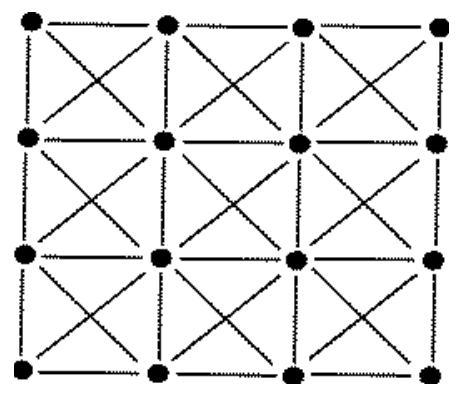


2. Séquence défigée : certains points de fixité sont désactivés - coexistence d'un sens global et d'un sens littéral :

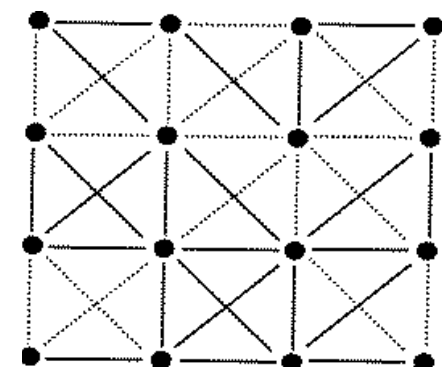

3. Séquence défigée : tous les points de fixité sont désactivés - le cas du défigement sémantique :

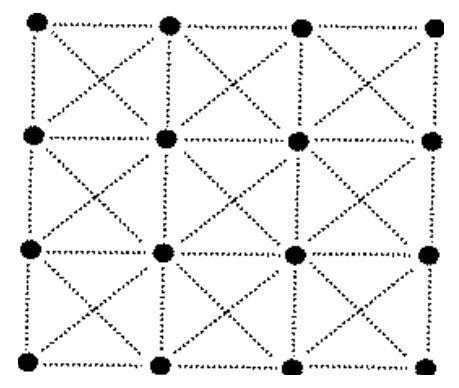

Ce dernier cas confirme la primauté du sens sur le reste des composants. Si une SF est défigée sémantiquement, même si les structures syntaxiques, rythmiques, lexicales, etc., restent apparemment intactes, tous les points de fixité sont nécessairement désactivés. Il y a donc une hiérarchie entre les niveaux : les uns sont plus englobants que d'autres.

\section{Pour ne pas conclure}

Nous n'avons évoqué là que quelques éléments essentiels de la problématique du figement que nous pouvons résumer comme suit :

— le défigement est loin d'être un fait marginal ; il est au cœur de la problématique du figement ;

— son étude a un impact heuristique certain : il permet entre autres de découvrir les mécanismes du figement ;

— ce qui contribue à ne plus voir dans le figement uniquement des structures syntaxiques (tournures, moules) ou des faits lexicaux (des mots) mais à le considérer comme un phénomène complexe impliquant toutes les dimensions linguistiques : prosodie, phonologie, morphologie, lexique, syntaxe, pragmatique, etc. ;

- le tout peut être représenté sous forme de trames comportant des points de fixité relatifs aux niveaux d'analyse ;

- le défigement serait ainsi appréhendé en termes de désactivation de ces points, désactivation qui pourrait être partielle ou totale ;

— d'où la notion de degré de défigement, dont l'ultime aboutissement est la 
combinatoire libre correspondant aux éléments constitutifs de la séquence figée de départ ;

— cette manière de voir a l'avantage de sortir le débat sur le figement et le défigement de la dualité globalité/littérarité et de souligner la complexité du phénomène qui ne peut être abordée indépendamment des facteurs contextuels et discursifs.

Il reste évidemment des questions en suspens. Nous nous n'en mentionnons que les trois suivantes :

— partant de ces éléments d'analyse, serait-il possible d'établir une typologie des défigements comme c'est le cas pour le figement?

- comme le défigement ne touche pas uniquement les séquences figées et qu'il les dépasse pour englober toutes sortes d'unités complexes comme les collocations, les apophtegmes, les citations et les maximes, n'y aurait-il pas là une autre façon de reprendre les critères du figement, ou, ne faudrait-il pas au contraire, avoir une conception du défigement plus restreinte?

- si la fixité et la congruence semblent être les deux notions qui gouvernent figement et défigement, ne serait-il pas souhaitable de leur en donner un statut théorique plus précis?

\section{Bibliographie}

ANDRE, C., 2011, Les états d'âmes. Un apprentissage de la sérénité, Odile Jacob, poche, p. 172.

ANSCOMBRE, J.-C., 2011, «Figement, idiomaticité et matrices lexicale », Le figement linguistique : la parole entravée, Honoré Champion, Paris, p. 17-40.

Balzac, H. de, Mémoires de deux jeunes mariés, Vol. 9, Classiques Garnier, p. 27.

- Un début dans la vie, Vol. 9, Classiques Garnier, p. 498.

Ben A mor B En HamidA, T., 2007, Les jeux de mots chez Raymond Queneau, Publications de la Faculté des Lettres et des Sciences Humaines de Sousse.

BEN JELlOUN, T., 2012, Le bonheur conjugal, Gallimard.

CÉLINE, L.-F., 1952, Voyage au bout de la nuit, Gallimard, Folio.

DAC, P., Avec mes meilleures pensées, Le Cherche midi, 2010.

FOUCAUlT, B. de, 1988, Les structures linguistiques de la genèse des jeux de mots, Peter Lang.

FREUD, S., 1905, Le mot d'esprit et ses rapports avec l'inconscient (Reed. 1971), Gallimard. 
GIRY-SCHNEIDER, 1987, Les prédicats nominaux en français. Les phrases simples à verbes supports, Droz.

Greciano, G., 1983, Signification et dénotation en allemand. La sémantique des expressions idiomatiques, Recherches linguistiques, Metz.

Gross, G., 1996, Les expressions figées en français, Ophrys, Paris.

KLEIBER, G., à paraître, «Proverbes et dictons : la dénomination, quel surplus sémantique? ", La phraséologie entre fixité et congruence, Hommages à S. Mejri, P.A. Buvet et I. Sfar (dirs.), Academia Bruylant, Sciences du langage : carrefours et points de vue.

LANZMANN, C., 2009, Le lièvre de Patagonie, Gallimard.

Le ClezIO, J.-M. G., 2011, Histoire du pied et autres fantaisies, Gallimard.

MEJRI, S., (dir.), 2008, Equivalences 35/1-2, Jeux de mots et traduction, Editions du Hazard, Bruxelles.

- 2008, «La traduction des jeux de mots », Equivalences 35/1-2, p. 71-83, Editions du Hazard, Bruxelles.

- (dir.) 2008, La traduction des séquences figées, META, Vol 53, n² 2, Les Presses de l'Université de Montréal.

— 2011, "L'opacité des séquences figées », Au commencement était le verbe. Syntaxe, sémantique et cognition, Mélanges en l'honneur du Prof. J. François, F. Neveu, P. Blumenthal et N. Lequerler (eds), p. 373-386, Peter Lang.

PENNAC, D., 2012, Journal d'un corps, Gallimard.

PERRIN, L., (à paraître), « Du figement au défigement des unités lexicales », Cahiers de lexicologie, Editions Garnier.

— 2011, "Figement, énonciation et lexicalisation citative », Le figement linguistique : la parole entravée, Honoré Champion, Paris, p. 81-94.

Rey, A., et Chantereau, S., 1989, Dictionnaire des expressions et locutions, Les Usuels du Robert.

Rossi, M. et PETER-DEFARE, E., 1998, Les lapsus : ou pourquoi ma fourche a langué, PUF, Paris.

SFAR, I., 2008, « Traduire les blagues : jouer par/avec les mots », Equivalences 35/1-2, p. 85-101, Editions du Hazard, Bruxelles.

SouteT, O., 2010, «Et que je te miaule..., En hommage à R. Martin, savant austère et amoureux des chats », Les configurations du sens, Franz Steiner Verlag Stuttgart, P. Blumenthal et S. Mejri (dirs.), p. 132-152.

VINCENT, J.-D., 2007, Voyage extraordinaire au centre du cerveau, Odile Jacob.

Vittoz CANuto, M.-B., 1983, Si vous avez votre jeu de mots a dire : analyse de jeux de mots dans la presse et dans la publicité, NIZET G., Paris. 